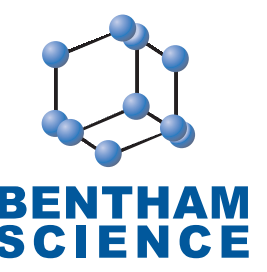

\title{
Alzheimer's Disease and Molecular Chaperones: Current Knowledge and the Future of Chaperonotherapy
} Antonella Marino Gammazza
and Alberto J.L. Macario ${ }^{2,3 *}$

\begin{abstract}
${ }^{I}$ Department of Biomedicine and Clinical Neurosciences, Human Anatomy Section, University of Palermo, Palermo, Italy; ${ }^{2}$ Euro-Mediterranean Institute of Science and Technology (IEMEST), Palermo, Italy; ${ }^{3}$ Department of Microbiology and Immunology, School of Medicine, University of Maryland at Baltimore; and IMET, Columbus Center, Baltimore, Maryland 21202, USA
\end{abstract}

\begin{abstract}
Background: Alzheimer's disease (AD) is a dementia, a neurodegenerative condition, and a protein-misfolding disease or proteinopathy, characterized by protein deposits, extracellular plaques and intracellular neurofibrillary tangles, which contain the AD's typical pathological proteins, abnormal $\beta$-amyloid and hyperphosphorylated tau, respectively, and are located predominantly in the cortex of the frontal, parietal, and temporal brain lobes. What is the role of molecular chaperones in AD? Data indicate that molecular chaperones, also known as Hsp, are involved in $\mathrm{AD}$, probably displaying protective roles and/or acting as pathogenic factors as it occurs in chaperonopathies in which case AD would be suitable to chaperonotherapy. Hsp60, Hsp70, and Hsp90 can be augmented and overexpressed or diminished and downregulated in various situations in AD affected tissues and cells, indicating they are active during disease development and progression. Question: What is the role of molecular chaperones in AD? Data indicate that molecular chaperones, also known as Hsp, are involved in $\mathrm{AD}$, probably displaying protective roles and/or acting as Objective: Investigate the role of Hsp in AD, focusing on Hsp60, Hsp70, and Hsp90. Method: Critical examination of published data. Results: Hsp60, Hsp70, and Hsp90 can be augmented and overexpressed or diminished and downregulated in various situations in $\mathrm{AD}$ affected tissues and cells, indicating they are active during disease development and progression. Conclusion and Perspectives: Notwithstanding that the roles and mechanisms of action of chaperones in $\mathrm{AD}$ are still incompletely understood, there is already enough evidence to encourage the development of therapeutic strategies targeting them, either to block their activity in case they promote disease progression or to boost their performance when they are protective. The latter is an example of positive chaperonotherapy, which also includes chaperone replacement via gene or protein administration. On the contrary, if a chaperone is found to help the disease, it has to be blocked or eliminated, which constitute modalities of negative chaperonotherapy.
\end{abstract}

Keywords: Chaperonopathies, protein-misfolding diseases, Alzheimer's disease, $\beta$-amyloid, tau, molecular chaperones, chaperonotherapy.

\section{INTRODUCTION}

Alzheimer's disease (AD) is a form of dementia. The loss of mental power that includes, depending on clinical stage, loss of memory; diminished capacity to understand complex concepts and face daily problems and solve them rapidly; impaired ability for communication; poor judgment; disorientation; confusion; behavioral changes and other psychological and motor abnormalities; is called dementia [1-3]. The causes of dementia are varied, for example, stroke with ischemic encephalopathy (multi-infarct or vascular dementia); head trauma; hydrocephalus; encephalitis; lysosome storage disease; chronic alcoholism; demyelinative conditions such as multiple sclerosis; and neurodegenerative diseases such as AD, Parkinson's and Huntington's diseases, Lewy body dementia, and others.

$\mathrm{AD}$ is a neurodegenerative condition. Classification of neurodegenerative diseases is still a project in progress and currently includes data from epidemiology, molecular biology, molecular genetics, and genetics [4-6]. However, the classification based on the predominant anatomic location of the distinctive pathological

*Address correspondence to this author at the School of Medicine, University of Maryland at Baltimore, IMET, Columbus Center, 701 E. Pratt St., Baltimore, MD 21202, USA; Tel/Fax: 240-631-9594;

E-mail: Ajlmacario@som.umaryland.edu proteins and lesions continues to be useful even today in practice, in clinics and clinical pathology. Consequently, one may speak of degenerative diseases affecting predominantly the gray matter, or the basal ganglia and nigrostriatal system, or the motor neurons, or the spinocerebellar system, among others [5, 7]. AD falls within the gray matter group, since the most affected structures are the cortex of the frontal, temporal, and parietal lobes; there is also some participation of the hippocampus.

$\mathrm{AD}$ is nowadays considered a member of the protein misfolding diseases [8-10]. These pathologic conditions have protein deposits, i.e., protein aggregates-precipitates, as one of the distinctive characteristics, and are considered proteinopathies. A protein is altered due to mutation or aberrant post-translational modification, namely it is a pathological form of a normal protein, and tends to form reversible aggregates and irreversible precipitates. These pathological molecules and the multimolecular deposits they form may be toxic to the cell, or may at least constitute a burden to the cell and interfere with its normal physiology. These conditions may be considered primary proteinopathies and $\mathrm{AD}$ is one of them.

Secondary proteinopathies are due to failure of the chaperoning system, which leads to multimolecular deposits (reversible aggregates and irreversible precipitates) of normal proteins and, even more so, of pathological proteins with tendency to aggregate. Whether this is the case for AD it is still unclear. Some data seem to 
Table 1. Examples of histopathological findings in AD specimens in comparison with findings pertaining to another gray matter disease $^{\mathrm{a}}$

\begin{tabular}{|c|c|c|}
\hline Disease & Histopathology & Proteins(s) $^{\mathbf{b}}$ \\
\hline \hline Alzheimer's (AD) & $\begin{array}{c}\text { Diffuse plaques; neuritic plaques; neurofibrillary tangles; } \\
\text { amyloid angiopathy; Hirano bodies (with actin); vacuoles } \\
\text { with argyrophilic granule }\end{array}$ & $\begin{array}{c}\text { APP; A } \beta \text {; tau; presenilin 1; presenilin 2; apolipoprotein E; } \alpha 2- \\
\text { macroglobulin; caveolin; complement regulators; ubiquitin; Hsp }\end{array}$ \\
\hline Pick's (PD) & Pick cells with Pick bodies & A $\beta ;$ tau \\
\hline
\end{tabular}

${ }^{\mathrm{a}}$ Source: Ref. [7]. See also ref. [13].

${ }^{\mathrm{b}}$ Proteins (and genes) normal or abnormal, known or suspected to play a role in pathogenesis. Abbreviations are: APP, amyloid precursor protein; A $\beta$, amyloid beta.

indicate that deficiencies in the chaperoning system, particularly in the protein-degradation arm with participation of the ubiquitinproteasome and chaperone-mediated autophagy machineries, may be involved in $\mathrm{AD}$ [11-13]. However, there is no definitive evidence that a failure of the chaperoning system by itself contributes in a determinant fashion to the pathogenic mechanism of $\mathrm{AD}$ [14]. In any case, the information already available encourages the development of chaperonotherapy for $\mathrm{AD}$, as discussed later.

As a typical proteinopathy, $\mathrm{AD}$ is characterized by abnormal proteins with mutations or post-translational modifications that interfere with their normal functions and status (soluble or not) and metabolism inside the cell [15-17]. Mutant proteins are the amyloid precursor protein (APP) and presinilins 1 and 2 (PS1 and PS2). In addition, the pathological, post-translationally modified protein in $\mathrm{AD}$ is the microtubule associated protein tau, which is hyperphosphorylated. These alterations, mutation and post-translational modifications, make the pathological proteins prone to aggregate and precipitate and, thereby, form the extracellular neuritic plaques and the intracellular tangles. While in the plaques abnormal forms of APP (i.e., $\beta$-amyloid produced by abnormal cleavage APP) predominate, in the tangles tau is the main component [18-20].

The molecular composition of the plaques and tangles has been investigated since many years ago in attempts to gain insight into the mechanisms that lead to their formation [18-23]. These studies illustrate the real power of histopathology at various levels of resolution (e.g., optical and electron microscopies), including complementary methods (immunohistochemistry and Western blotting, among others) to dissect complex histological bodies, plaques and tangles in the case of $\mathrm{AD}$, into their molecular components. The results of this multipronged histopathological approach are summarized in Table $\mathbf{1}[7,13]$.

Many but no all Hsp are chaperones and there are chaperones that are not Hsp. Nonetheless, the terms Hsp and chaperones are used indiscriminately in the literature and we will use them as synonyms in this article. It has been realized that the chaperones constitute a physiological system distributed throughout the body and its components are present in all cellular compartments as well as extracellularly [14]. In addition, many conditions have been identified in which one or more components of the chaperoning system are abnormal, quantitatively and/or qualitatively, and are at the basis of pathogenic mechanisms that lead to manifest cell and tissue pathology and disease. These diseases, in which abnormal chaperones play an etiologic-pathogenic role, are the chaperonopathies [24]. The question is: are Hsp-chaperones quantitatively (elevated or decreased) and/or qualitatively (e.g., posttranslationally modified, or mutated) abnormal in AD-affected cells and tissues, e.g., in plaques and tangles? Namely, are there signs indicating that AD is a chaperonopathy? Data in Table 1 show that Hsp-chaperones are present in the affected brain tissue, but their possible structural abnormalities (e.g., post-translational modifications) and their pathogenetic or protective roles in $\mathrm{AD}$ are still under study. Clarification of this issue will have an impact on how AD is approached clinically and therapeutically. For example, it may be found that a given chaperone is a good indicator of disease status and, thus, this chaperone will become a biomarker worth measuring periodically in the patient's follow up. If, in addition, it is established that a given chaperone actively contributes to disease initiation and/or progression one may think of ways to eliminate or block the pathogenic chaperone. Likewise, if a chaperone malfunction or functional failure is identified as cause of pathology, strategies and means to replace the defective chaperones will have to be developed. In other words, if $\mathrm{AD}$ is found to be a chaperonopathy, it will become clear that it is a condition amenable to chaperonotherapy, positive when chaperone replacement or boosting is to be implemented, or negative, when the pathogenic chaperone is to be blocked or eliminated [25-27].

Where to look for chaperones in $\mathrm{AD}$ and what to look for? Since we now know that chaperones can reside and function extracellularly as well as intracellularly, and that their quantity and distribution changes in many pathological processes, one of the first objectives would be to determine if there are quantitative variations of chaperones around and inside the various cell types in the affected brain tissue, including plaques and tangles. On this, there is some information already, which will be discussed later.

What chaperones to look for in AD? There is a large variety of chaperones, and those that have been found to cause chaperonopathies are displayed in Table 2. Since mitochondria are affected in $\mathrm{AD}$, to the point that for some authors $\mathrm{AD}$ is a mitochondriopathy $[28,29]$, and considering that the mitochondria unfolded protein response (mtUPR) is activated in AD [30], chaperones known to reside and work in mitochondria should be preferentially targeted for analysis. In this regard, Hsp60 and Hsp10 are the pertinent candidates, and the information currently available about them is discussed later.

On the other hand, considering that $\mathrm{AD}$ is a proteinopathy, with accumulation of misfolded proteins, which are known to trigger the unfolded protein response (UPR) with major involvement of the endoplasmic reticulum (ER) [31], one may target for analysis those chaperones that work in the ER, for example GRP78/BiP, GRP94/ gp96, GRP170/ORP150, GRP58/ERp57, PDI, calnexin, calreticulin, and the co-chaperone SIL1, just to name some candidates for study. The available information about some of them in what pertains to $\mathrm{AD}$ is discussed later.

\section{ALZHEIMER'S DISEASE, CELLULAR STRESS, AND CHAPERONES}

$\mathrm{AD}$ is the most common form of dementia and is characterized by a rapid progression from episodic memory deficits to a decline 
Table 2. Subpopulations of Hsp-chaperones by molecular weight found to cause chaperonopathies ${ }^{\mathrm{a}}$

\begin{tabular}{|c|c|c|}
\hline kDa & Classical family & Others causing chaperonopathies \\
\hline$\geq 200$ & None & Sacsin \\
\hline $100-199$ & Hsp100 - 110 & $\mathrm{VCP}$ \\
\hline $81-99$ & Hsp90 & Paraplegin [SPG7]; UNC45B \\
\hline $65-80$ & Hsp70/DnaK & Spastin [SPG4] \\
\hline $55-64$ & $\begin{array}{l}\text { Hsp60 (chaperonins Groups I } \\
\text { and II, e.g., Cpn60 and CCT- } \\
\text { BBS }\end{array}$ & $\begin{array}{l}\text { BAG3; immunophilins FKB-type: peptidyl-prolyl cis-trans isomerases (PPI; FKBP5; FKBP10); } \\
\text { myocilin; protein disulfide-isomerases (PDI; ER); SERPINH1 (Hsp47) }\end{array}$ \\
\hline $35-54$ & Hsp40/DnaJ & $\begin{array}{l}\text { AIP; AIPL1; BCS1L; CALR; clusterin; DNAJC19; FOXRED1; melusin; morgana; SIL1; TBCE; } \\
\text { torsin A }\end{array}$ \\
\hline$\leq 34$ & sHsp (crystallins) & $\begin{array}{l}\text { Alpha Hemoglobin-Stabilizing Protein (AHSP); alpha-synuclein; BCAP31; frataxin; Hsp10 } \\
\text { (Cpn10); HSPB11; immunophilin cyclophilin-type: peptidyl-prolyl cis-trans isomerase (PPI; } \\
\text { PPIB); LRPAP1; RP2; SOD1-2 }\end{array}$ \\
\hline
\end{tabular}

${ }^{\mathrm{a}}$ Source: Ref. [14].

in overall cognitive functions, impairing the patients' ability to carry out activities of daily living with death occurring usually within 10 years after diagnosis [3]. In 2005, AD International convened a multi-national panel of dementia experts to work on an evidence-based consensus on dementia prevalence worldwide. The panel estimated that there were 24.3 million people with dementia in the world in 2001, and predicted that this would rise to 42.3 million in 2020, and to 81.1 million by 2040 [32].

From a pathological point of view, AD is characterized by the cortical presence of extracellular deposits of amyloid- $\beta$ (A $\beta$, Abeta), called senile or neuritic plaques, and intraneuronal inclusions, called neurofibrillary tangles (NFTs) formed by accumulation of abnormal filaments of tau, both found predominately in the brain regions involved in memory and learning [13, 18-23].

There are currently several research efforts aimed at delaying the onset of $\mathrm{AD}$ and the potential cytoprotective effects of the heat shock response are an attractive target for pharmacological therapies [27]. This is particularly relevant for neurodegenerative diseases associated with protein misfolding and subsequent aggregation such as $\mathrm{AD}[13,33,34]$. Molecular chaperones, many of which are Hsp as mentioned earlier, are the main components of the chaperoning system and play a variety of roles in addition to maintaining protein homeostasis. Hsps can be overexpressed as a means for protection against stressors such as heat, oxidative stress, and ischemia. Hsp-chaperones mediate the refolding or degradation of stress-damaged proteins, depending on whether the damage is reversible or not and, thus, protect the cells from potential deleterious effects of unfolded-damaged proteins and promote the cell's resistance to, and recovery from, stress, all events pertinent to AD [33]. Besides, Hsps have many other functions inside and outside cells; for instance, they play roles in cell division, apoptosis inhibition, and metastasis of cancer cells [14].
As mentioned earlier, many neurodegenerative diseases, including $\mathrm{AD}$, are characterized by the accumulation of aberrantly folded protein species [8-10], but it is not yet clear whether failure of the chaperoning system contributes to the pathogenic mechanism of AD. In other words, thus far there is no conclusive evidence that $\mathrm{AD}$ is a primary chaperonopathy although various observations indicate that this is a real possibility for at least some specific cases.

Chaperonopathies have been classified considering quantitative and qualitative features into by defect, by excess, and by mistake [35]. This classification is useful to establish a diagnosis with physiopathological implications and, thus, aids in decisions on treatment. Further, the classification is instrumental to conduct a differential diagnosis exercise that will lead to an accurate identification of syndrome and disease, which is key to proper patient management.

Another way of classifying chaperonopathies that also helps the physician and, therefore, the patient, is based on whether they are inherited (genetic) or acquired [14]. The pathogenic chaperone may be normal or structurally altered and belong to any of the known Hsp-chaperones groups (Table 2). The structural alteration of a pathological chaperone may affect any of its functional domains or modules. When the chaperone is deficient, the aim is to replace it or to boost its functionality, and we can refer to this therapeutic modality as positive chaperonotherapy. If, on the contrary, the chaperone favors disease rather than protecting the cell and the organism, the therapeutic strategy aims at eliminating or blocking the pathological chaperone. This latter therapeutic modality may be referred to as negative chaperonotherapy $[25,27]$.

Involvement of chaperones in the pathogenesis of $\mathrm{AD}$ was first proposed following promoter analysis of the amyloid precursor protein (APP) gene, where a heat shock element (HSE) was found within its promoter [36]. As mentioned earlier, molecular chaperones play essential roles in many cellular processes including pro- 
tein folding, translocation across membranes, refolding, and degradation, and also in disaggregation of protein aggregates (see references earlier). This explains why molecular chaperones are essential in the cellular defence against protein aggregation caused by misfolding both at intra- and extra-cellular levels and are potentially powerful suppressors of neurodegeneration. Moreover, chaperones regulate protein functions in order to protect against the oxidative stress accompanying the toxicity of amyloid aggregates and their expression is increased under conditions of amyloid challenge [37]. Also, a "sink hypothesis" has been proposed, positing that cellular toxicity and other manifestations of chaperone deficiency in $\mathrm{AD}$, and other similar conditions, may develop because chaperones are being sequestered on the amyloid fibrils, as well as on the other protein precipitates and, thus, are re-directed away from their normal cellular tasks [38]. This situation can be described as a chaperonopathy by defect due to a quantitative deficit of chaperones at the time and place, i.e., where and when, they are needed [14]. Here, we discuss growing evidence suggesting a convergence between $\mathrm{AD}$ and molecular chaperones and the possibility that this disease may be a chaperonopathy. This approach provides a convenient point of view to examine $\mathrm{AD}$ and to look for new therapeutic avenues. In this chapter, we focus on Hsp60, Hsp70, and Hsp90, discussing some of their biological, molecular, and pathological facets as they pertain to AD.

\subsection{Hsp60}

Hsp60 is classically described as a mitochondrial protein whose gene is induced by various types of stressors as heat shock, oxidative stress, and DNA damage [39]. Inside mitochondria, Hsp60 acts as a folding machine, together with Hsp10, for the correct folding of several mitochondrial proteins $[40,41]$. Various studies, published over the past several years, have demonstrated new subcellular locations and functions for Hsp60, describing it as a ubiquitous molecule with multiple roles in health and disease [42-44]. Moreover, detailed studies of the structure of Hsp60 have been reported that help understand the functions of this multifaceted molecule $[45,46]$.

Hsp60 has both pro-survival and pro-death functions depending on tissue, cell type, and apoptosis inducers [47, 48]. It has been demonstrated that Hsp60 may associate with Bak or Bcl-XL in normal heart tissue, inhibiting their apoptotic potentials and consequent cytochrome c release [49]. Hsp60 may influence tumor progression as suggested by its accumulation in the cytosol and plasma membrane of cancerous cells, reaching the extracellular space via secretory vesicles that in turn can modulate (induce, or inhibit?) anti-tumor immune responses [50-52]. Hyperacetylation of Hsp60 in osteosarcoma cells is associated with the anticancer activity of geldanamycin and Hsp60 nitration is associated with the anti-tumor action of the histone deacetylase inhibitor SAHA [53, 54]. These findings support the idea that Hsp60 is a suitable target for antitumor therapy and opens new prospects for novel drugs design [55], a concept that can be extended to the developing of agents to treat AD.

On the other hand, there exists published evidence supporting a cell death-promoting function for Hsp60. Mitochondrial Hsp60 binds pro-caspase 3 in Jurkat and Hela cells, accelerating the maturation of pro-caspase 3 by upstream activator caspases during apoptosis $[56,57]$.

Hsp60 has also been implicated in the mechanism of cell aging [58]. Senescent cells in culture become flat and enlarged and can be maintained in a viable state for long periods, but cannot be induced to divide by mitogenic stimuli [59]. Senescence-induced resistance to apoptosis leads to an increase in the number of senescent cells inside tissues, with consequences on the feebleness of tissue integrity and on neoplastic transformation, likely promoting the development of late-life cancers [60]. During replicative senescence of normal human skin fibroblasts, the levels of Hsp60 increase and form a complex with MOK, a MAP kinase involved in signal transduction to the nucleus [61]. Furthermore, a correlation between increased levels of Hsp60 and senescence of skin fibroblasts was shown to involve interaction between Hsp60 and mtHsp70 [62].

Hsp60 may be involved in autoimmune processes: it may act as autoantigen because of the high sequence similarity (molecular mimicry) between human and foreign Hsp60 from bacteria and parasites that colonize humans, which leads to anti-Hsp60 antibody cross-reactivity $[14,63]$. Hsp60 has high structural similarity also with human proteins such as myelin-associated protein, glutamic acid decarboxylase, and acetylcholine receptor, further suggesting that circulating Hsp60 may serve as autoantigen for the generation of an autoimmune response [14]. Consequently, Hsp60 may trigger autoimmune pathological conditions, including some affecting the nervous system such as multiple sclerosis and myasthenia gravis $[14,64]$.

Extracellular Hsp60 can interact with a variety of receptors present on the plasma-cell membrane surface, such as CD14, CD40, CD91 and TLRs $[65,66]$. Hsp60 influences the pro-inflammatory capacity of adipocytes binding an adipocyte receptor and contributing to obesity-associated inflammatory disease leading to diabetes [67]. The chaperonin has also been implicated in the pathogenesis of chronic inflammatory conditions such as Crohn's disease [68], ulcerative colitis $[69,70]$, alcoholic liver disease [71], chronic obstructive pulmonary disease [72], and atherosclerosis [73].

Neural expression of Hsp60 increases over the course of development, a trend consistent with the changes of mitochondrial content in the brain [74]. In brain, Hsp60 is endogenously expressed in astrocytes, neurons, microglia, oligodendrocytes, and ependymal cells [74]. This distribution suggests an active participation of this chaperonin in many functions of the brain, both in normal and pathological conditions. Hsp60 exposed on the surface of astrocytes and neuroblastoma cells interacts with TREM2, a receptor that, if mutated, is responsible for genetic disorders affecting bones and brain [75]. Extracellular Hsp60, through binding to LOX-1 (lectinlike oxidized low-density lipoprotein receptor-1), may promote microglia-mediated neuroinflammation and a prolonged delay in the induction of Hsp60 in injured regions of the brain [76, 77]. For example, expression of Hsp60 has been found increased in the brainstem after subarachnoidal haemorrhage, forebrain or focal cerebral ischemia, and neonatal hypoxia-ischemia [77]. Increased levels of the chaperonin have also been found in both animals and patients with temporal lobe epilepsy in response to epileptic seizures [78].

Given that Hsp60 plays a critical role in assisting the correct folding of other mitochondrial proteins and enzymes, a deficiency in its concentration and/or function together with the increased vulnerability to oxidative stress observed in the tissues of neurodegenerative disorders, may lead to severe conditions caused by protein misfolding and aggregation $[24,79]$. This would be an Hsp60 chaperonopathy.

Post-translational modifications (phosphorylation, acetylation, Y-nitration, S-nitrosylation, methylation, oxidation, and ubiquitination) of Hsp60 may occur through a variety of mechanisms, resulting in the loss or impairment of its functions [54, 80]. Such Hsp60 deficiency might be a common cause of mitochondrial dysfunction, which would be significant since $\mathrm{AD}$ has been described as a disorder aggravated by oxidative stress and/or mitochondrial defect characterized by protein conformation abnormalities (Fig. 1) [29, $30,77,81]$.

Hsp60 was found to be involved in preventing aggregation by trapping misfolded forms of prion protein scrapie ant thus preventing glycation, which is known to contribute to the pathogenesis of protein deposition [82]. Expression of Hsp60 is significantly decreased in the parietal cortex of AD subjects and in the cerebella of 


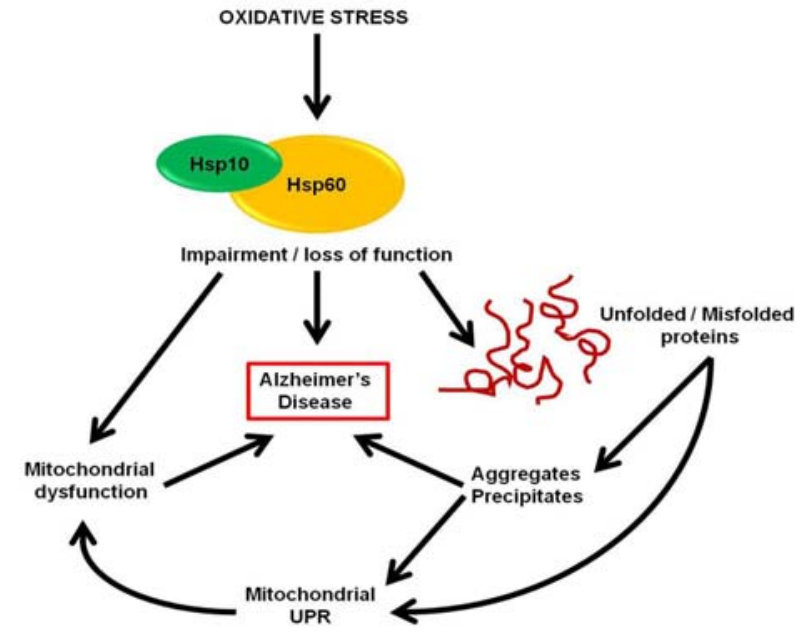

Fig. (1). Stress and Hsp60 in Alzheimer's disease: possible pathogenic pathways. Oxidative stress (and other types of stress) may cause Hsp60 alterations, e.g., post-translational modifications, resulting in an impairment or loss of the chaperonin's functions with various consequences (some of which are indicated in the figure) and, thus, contributing to the development and/or progression of Alzheimer's disease. UPR, unfolded protein response.

a rat model of $\mathrm{AD}$, suggesting a defect in the protective role of this chaperonin in the AD brain $[81,83]$. In support of the neuroprotective effects of Hsp60, it has been demonstrated that in a human neuroblastoma cell line, induced expression of the chaperonin prevented intracellular $\beta$-amyloid-induced inhibition of complex IV and consequently reduced apoptosis [84]. A $\beta 25-35$ induced oxidation of Hsp60 in fibroblasts derived from AD patients [85] and, also, Hsp60 was oxidized by A $\beta 1-42$ leading to a loss of function of the chaperonin, which caused an increase in protein misfolding and aggregation [86]. Again, this would be a chaperonopathy by defect.

Hsp60 levels were found elevated in lymphocytes from $A D$ patients when compared to controls [87] and sporadic AD subjects exhibited a significant ( $40-60 \%)$ increase in expression levels of genes activated by the mitochondrial unfolded protein response (mtUPR), including genes encoding mitochondrial chaperones such as Hsp60 and others [30].

Administration of an $A \beta$ amyloid-Hsp60 peptide-conjugate vaccine led to the induction of anti-A $\beta$-specific antibodies, associated with a significant reduction of cerebral amyloid burden and of the accompanying inflammatory response in the brain of a mouse model of AD [88]. On the contrary, other authors have attributed a deleterious effect to the elevated expression of Hsp60 in AD. For example, it was shown that Hsp60 mediates in vitro the translocation of APP to the mitochondria leading to dysfunction of this organelle [89].

All the findings discussed in the preceding paragraphs indicate that more research is needed to determine with certainty if $\mathrm{AD}$ is an Hsp60 chaperonopathy by defect, or by excess, or by mistake. However, the data do show that this chaperonin is implicated in the disease and deserves more research to clarify what it does, when, and how, as a fundamental preliminary step to the developing of specific Hsp60 chaperonotherapy, positive or negative, as the case might be.

\subsection{Hsp70}

The Hsp70 family is large with at least 17 members [90], some of which are stress-inducible (e.g., HSPA1A also named Hsp70i), while others are constitutively expressed (e.g., HSPA8 also called
Hsc70). The stress-inducible Hsp70 proteins are among the first to be upregulated upon heat shock to cope with the protein damagemisfolding caused by stress. Hsp70 chaperones are found in most cellular compartments, including the nucleus and cytoplasm (Hsc70), mitochondria (mtHsp70, also named HSPA9 and mortalin), and ER (Grp78, also known as BiP) [90, 91].

Hsc70 assists the folding of client proteins via an ATPdependent mechanism binding to short extended hydrophobic regions of the misfolded client proteins and, thereby, prevents aggregation of the unfolded clients [92]. By differentially associating with the co-chaperones Hsp40, and with Hsp90, Hsp70 achieves versatility of function in the various cellular compartments [93].

Hsp70 inhibits apoptosis acting on the caspase-dependent pathway at several steps both upstream and downstream of caspase activation and, also, on the caspase-independent pathway. A study of the correlation between the anti-apoptotic effects of Hsp70 and inhibition of caspase activity suggested that the point of Hsp70 intervention was upstream of caspase-3 activation. Subsequently, it was demonstrated that Hsp70 is able to directly inhibit caspase processing by interacting with Apaf-1 and prevent the recruitment of procaspase-9 to the apoptosome [94]. Overproduction of Hsp70 leads to increased resistance against apoptosis-inducing agents such as tumor necrosis factor-a (TNFa), staurosporin, and doxorubicin, while downregulation of Hsp70 levels by siRNA leads to increased sensitivity towards these agents [95-97]. In various kinds of tumors increased Hsp70 levels are observed that correlate with increased malignancy and resistance to therapy [98]. For example, Hsp70 binds tumor-suppressor proteins, such as p53 and HER2, and thus allows unlimited cellular growth and increased resistance to chemotherapy in breast cancer, consequently, elevated levels of Hsp70 are associated with poor prognosis in breast and endometrial cancers [98]. Downregulation of the Hsp70 levels in some types of cancers induce differentiation and cell death [99].

Hsp70 has the ability to trigger the activation of the immune response by stimulating both innate and adaptive immunities. The immunogenicity of Hsp70 and its capacity to activate antigenspecific immunity have made this protein a promising candidate for vaccine development. With respect to innate immunity, Hsp70 is secreted from host cells into the extracellular milieu, and this extracellular Hsp70 triggers innate immunity via activation of antigen presenting cells (APCs) [100]. Regarding adaptive immunity, Hsp70 is an abundant antigen for both B and T cells. For example, the $P$. falciparum $\mathrm{Hsp} 70$ is expressed by most parasites during their life cycles and it is recognized by serum antibodies from malaria patients [101].

The numerous roles of the various Hsp70 proteins relate not only to oncogenesis and the immune response, as discussed above, but also to other pathological processes, such as neurodegeneration and senescence. Neurodegenerative diseases such as AD, Parkinson's disease, Huntington's chorea, and spinocerebellar ataxias are characterized by excessive apoptosis. In some model systems, overexpression of Hsp70, or one of its co-chaperones, was shown to overcome the neurodegenerative symptoms induced by expression of a disease-related gene [102, 103].

Senescence in cell culture as well as aging in vivo is correlated with a continuous decline in the ability to mount a stress response [104]. Age-related symptoms and diseases reflect this decreased ability to cope with cellular stresses. Interestingly, centenarians seem to be an exception to the rule, as they show a significant induction of Hsp70 production after heat shock challenge [105]. In the brains of patients affected by $\mathrm{AD}$, an increased level in the expression of Hsp70 has been reported [106], suggesting a potential role of Hsp70 in the pathogenesis of this disease. APP interacts with the ER resident chaperone BiP/Grp78 during its normal processing in the ER-Golgi pathway, suggesting that an increase of 
Grp78 levels may help proper processing of APP therefore reduce amyloid production [107].

Immunohistochemical studies and expression analyses in $\mathrm{AD}$ brain tissue showed that expression levels of Hsp70 were elevated in affected regions, and this elevation appeared to be correlated to the presence of activated glia and dysregulated or stressed neurons [33]. The increase in Hsp70 levels could be related to an increase in the expression of the transforming growth factor $\beta 1$ (TGF-1), an enzyme that is thought to be responsible for the degradation of $A \beta$ [108]. In addition, cytosolic Hsp70 was shown to inhibit early stages of amyloid aggregation protecting against intracellular $A \beta$ [109]. It was reported that chaperone proteins, including Hsp70, can recognise abnormal tau and reduce its concentration by facilitating its degradation and de-phosphorylation [110-112]. Moreover, it has been demonstrated that tau binds directly to Hsp70, and Bag-1 has a role in this interaction [113]. Therapeutics that exploit the ability of Hsp70 to selectively target abnormal tau can rapidly and potently rescue the synaptic dysfunction that occurs in $\mathrm{AD}$ and other tauopathies [114].

It is clear from the above paragraphs that various members of the Hsp70 family are implicated in AD and it is also apparent that, as the pathogenic and/or protective mechanisms in which these chaperones (with or without their co-chaperones) participate are being elucidated, new perspectives for Hsp70 chaperonotherapy will be unveiled.

\subsection{Hsp90}

Hsp90, of which in humans there are at least five varieties (HSP90A in cytosol; HSP90alpha, HSP90beta, HSP90B (or Grp94) in the ER, and TRAP in mitochondria [14] is the most abundant protein in eukaryotic cells under unstressed conditions, and like other molecular chaperones, is present in one form or another in most cellular compartments such as the cytosol, endoplasmic reticulum, mitochondria, and chloroplast [115]. Hsp90 is indispensable for cell survival, playing an important role in the folding of many proteins pertaining to essential signalling pathways, and in the refolding of denatured proteins after stress $[115,116]$. Hsp90 is an ATP-dependent chaperone, with a unique N-terminal ATP binding site (in addition to another C-terminal nucleotide binding site) that has allowed for the development of specific Hsp90 inhibitors such as geldanamycin, a macrocyclic antitumor agent and its less toxic analog 17-allylamino-17-demethoxygeldanamycin (17AAG) [115]. Hsp90 does not bind to non-native proteins but rather to substrates in their native states and targets a specific set of client proteins that are involved in signal transduction [117].

Hsp90 has been shown to interact with important client kinases, including ErbB2, Src, Abl, and Met tyrosine kinases, and cyclindependent serine kinases [116], which are part of an intricate multidimensional signalling web that integrates information from various origins. Many of these client proteins are known to be bound to Hsp90 in an inactive state and are activated upon dissociation from Hsp90. For example, the catalytic domain of raf proteins, members of MAP kinase kinase kinases (MEKKs), are in a heterocomplex with Hsp90, and are activated upon dissociation from this Hsp90 such as in stress conditions [107]. Moreover, the abundant cytosolic Hsp90 under normal conditions has the capacity to bind to and keep stress transcription factors such as Heat Shock Factor-1 (HSF-1) in a monomeric inert state. Upon heat shock, HSF-1 is released from the Hsp90 complex due to the increased demand for chaperones by proteins in need of assistance for folding and refolding. This represents the first step in the activation of HSF-1 and the inducible Hsp90 offers a negative feedback loop to control the transcriptional activity of HSF-1 [107].

Hsp90 is overexpressed in cancer cells and is essential for the malignant transformation and progression of several tumor types such as bladder, breast and lung cancers, and leukemia [98, 118]; these being examples of chaperonopathies by mistake. Conse- quently, blockage of Hsp90 function in these cancers via inhibitory compounds may be a valuable therapeutic strategy [119], and by extension the same principles may be applied to the developing of compounds to treat AD if it is demonstrated that Hsp90 favors disease (AD would thus be an Hsp90 chaperonopathy by mistake).

Some observations suggest an additional role for Hsp90 in buffering some of the client proteins that might be directly responsible for disease progression $[120,121]$. Hsp90 was shown to inhibit early stages of amyloid aggregation [109] and administration of Hsp90 inhibitors to primary neurons prevented $A \beta$-induced neurotoxicity [122]. The complex of Hsp90 and Hsp70/Hsp40 also can inhibit $\mathrm{A} \beta$ formation and slow down the rate of aggregation in a chaperone concentration-dependent manner (Fig. 2) [123]. Hsp90 outside the cells may facilitate $A \beta$ clearance by the activation of microglial phagocytosis and $\mathrm{A} \beta$ degradation by NF- $\mathrm{KB}$ and $\mathrm{p} 38$ MAPK activation through the Toll-like receptor-4 (TLR4) pathway $[109,124]$. All these observations suggest that positive chaperonotherapy centred on Hsp90 could be a promising means to treat AD.

The other hallmark of the pathology of AD is the accumulation of tau, which leads to the formation of toxic NFTs. Chaperones are necessary to maintain tau in a non-aggregated state. Positive correlations have been found in the soluble protein levels from AD brain tissue between tau and molecular chaperones including Hsp90 [125]. Hsp70 and Hsp90 work together to coordinate tau homeostasis [126]. Pharmacologic inhibition of Hsp90 significantly reduced intracellular levels of the disease-associated phosphorylated tau species pS202/T205 and pS396/S404 (Fig. 2) [33]. These Hsp90 inhibitors primarily facilitate the clearance of phosphorylated tau via proteasomal degradation [33]. Mutant tau is maintained in tauopathies by Hsp90 and the inhibition of Hsp90 leads to reduction in the pathogenicity of these mutant species [121]. These data suggest that negative chaperonotherapy aimed at Hsp90 inhibition might offer hopes for developing efficacious treatment for AD.

Important regulators of the Hsp90 machinery are co-chaperones and posttranslational modifications, e.g., acetylation, nitrosylation, and phosphorylation, of the Hsp90 protein itself [115]. For instance, the acetylation of Hsp90 inhibits client binding thereby enhancing the proteasomal degradation of the unbound clients. Tau is a client for Hsp90 complexes. If tau is in an abnormal or modified form, then it can trigger the recruitment of CHIP, a cochaperone of Hsp90, to the complex, which induces the ubiquitination of tau and activates its downstream degradation steps [123].

Hsp90 inhibition may be suitable to a dual therapeutic approach in AD (Fig. 2). On the one hand, Hsp90 inhibitors may counteract tau protein hyper-phosphorylation and subsequent aggregation. On the other hand, Hsp90 inhibitors may reduce the protective stress response. Hsp90 can tightly bind and thus down-regulate the activity of HSF-1 but in the presence of cellular stress (a common event in AD), HSF-1 dissociates from Hsp90, translocates to the nucleus and binds heat-shock response elements, thereby inducing heat shock response genes, such as those encoding the proteins Hsp27, Hsp40, Hsp70, and Hsp90. The expression of these chaperone genes expands the buffering capacity of the cell and restores protein homeostasis under stressful conditions [127].

Another aspect to take into account in this context is that cell life and functions depend on many extracellular signals and that these signals, in turn, depend on carriers that travel communicating different cells. In the last years, growing evidence supports the pivotal role of the extracellular vesicles (EVs), such as exosomes, in cell-cell communication. The roles of exosomes in mediating the pathogenesis of $\mathrm{AD}$ were initially attributed to the capacity of these nanovesicles to serve as vehicles for the transport of the $A \beta$ peptides to the extracellular environment [128]. Recently, it has been demonstrated that Hsp70 and 90 intercellular transport via exosomes contributes to maintenance of protein homeostasis at the organismal level [129]. The data indicate that EVs are promising 


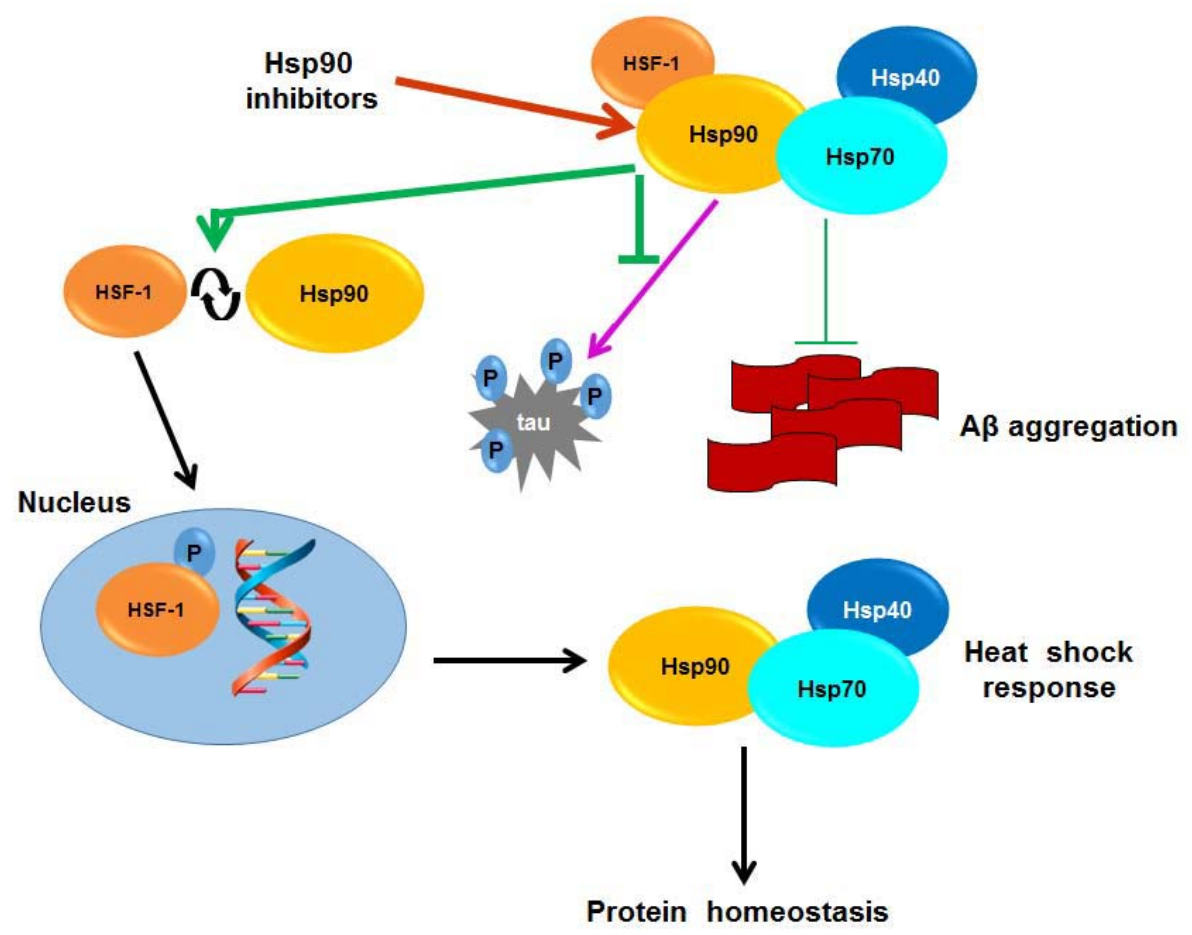

Fig. (2). Hsp90 inhibitors in Alzheimer' disease. Hsp90 can have positive (i.e., anti-pathogenic) and negative (i.e., pathogenic) effects in Alzheimer's disease as illustrated in the figure. For instance, positive effects consist of inhibiting $A \beta$ formation and slowing down the rate of aggregation mediated by the complex of Hsp90/Hsp70/Hsp40 (as indicated by the thin green T-shaped stick). Also, extracellular Hsp90 facilitates A $\beta$ clearance and A $\beta$ degradation. As a negative effect, Hsp90 promotes tau hyper-phosphorylation (P) and aggregation (as indicated by the violet arrow). In this context, the use of Hsp90 inhibitors (indicated with a red arrow) for treatment may involve a dual approach. The Hsp90 inhibitors may counteract tau hyper-phosphorylation and subsequent aggregation (as indicated by the thick green T-shaped stick), or may promote the protective stress response, as indicated by the long green arrow. The latter effect is due to the fact that Hsp90 can bind and down-regulate the activity of HSF-1 but in the presence of some Hsp90 inhibitors and cellular stress, HSF-1 dissociates from Hsp90 (as indicated by the two curved arrows), translocates to the nucleus and binds heat-shock response elements, inducing heat-shock response genes, such as those encoding Hsp40, Hsp70, and Hsp90, and restoring protein homeostasis.

elements for directing specific therapeutic agents to selected cells in which the chaperoning system needs re-enforcements or repair.

Since neurons are a terminally differentiated, i.e., they are a post-mitotic cell type, it has been suggested that neurons are especially susceptible to the cumulative effects of misfolded proteins as they are unable to reduce the load of toxic intermediates through consecutive rounds of mitosis [37]. Therefore, the capacity of neuronal chaperones to reduce misfolded proteins is essential for maintaining neuronal integrity. This again clearly points to positive chaperonotherapy as one promising treatment modality for AD.

\section{CONCLUSION AND PERSPECTIVES}

From the data discussed in the preceding three sections, we can say for Hsp60, Hsp70, and Hsp90 that there are clear indications of their active participation in the cellular and tissue phenomena typical of $\mathrm{AD}$, but the pertinent molecular mechanisms are still not fully understood. Nevertheless, there is growing evidence suggesting that the development of pathological features in AD could be the result of chaperone malfunctioning by excess, defect, or mistake. Consequently, AD is a candidate for chaperonotherapy in its various forms, positive, negative, and combined. These possibilities are currently under intense scrutiny in our laboratories and in those of many others, ensuring a bright future for research on this serious and widespread neurodegenerative condition and nurturing hope for those affected by it.

\section{CONFLICT OF INTEREST}

The authors confirm that this article content has no conflict of interest.

\section{ACKNOWLEDGEMENTS}

This work was supported by the Euro Mediterranean Institute of Science and Technology, and funded with the Italian National Operational Programme for Research and Competitiveness 2007-2013 grant awarded to the project titled "Cyber Brain - Polo di innovazione" (Project code: PONa3_00210, European Regional Development Fund). A.J.L.M. and E.C. de M. were partially supported by IMET; A.J.L.M. and F.C. were partially supported by IEMEST. This work was done under the umbrella of the agreement between the Euro-Mediterranean Institute of Science and Technology (Italy) and the Institute of Marine and Environmental Technology (USA) signed March 2012 (this is IMET contribution number IMET 16175).

\section{REFERENCES}

[1] Jellinger KA, Bancher C. Classification of dementias based on functional morphology. In: Jellinger $\mathrm{K}$, Ladurer $\mathrm{G}$, Windisch $\mathrm{M}$, Eds. New Trends in the Diagnosis and Therapy of Alzheimer's Disease (Key Topics in Brain Research). Vienna: Springer 1994; pp. 939

[2] Emre M. Classification and diagnosis of dementia: a mechanismbased \approach. Eur J Neurol 2009; 16: 168-73.

[3] Ballard C, Gauthier S, Corbett A, Brayne C, Aarsland D, Jones E. Alzheimer's disease. Lancet 2011; 377: 1019-31.

[4] McGeer PL, McGeer EG. NSAIDs and Alzheimer disease: epidemiological, animal model and clinical studies. Neurobiol Aging 2007; 28: 639-47.

[5] Armstrong RA. On the 'classification' of neurodegenerative disorders: discrete entities, overlap or continuum? Folia Neuropathol 2012; 50: 201-8. 
[6] Kovacs GG. Current concepts of neurodegenerative diseases. EMJ Neurol 2014; 1: 78-86.

[7] Macario AJL, Conway de Macario E. Molecular chaperones and age-related degenerative disorders. Adv Cell Aging Gerontol 2001; 7: 131-62.

[8] Selkoe DJ. Cell biology of protein misfolding: the examples of Alzheimer's and Parkinson's diseases. Nat Cell Biol 2004; 6: 105461 .

[9] Luheshi LM, Crowther DC, Dobson CM. Protein misfolding and disease: from the test tube to the organism. Curr Opin Chem Biol 2008; 12: 25-31.

[10] Bayer TA. Proteinopathies, a core concept for understanding and ultimately treating degenerative disorders? Eur Neuropsychopharmacol 2015; 25: 713-24.

[11] Ma QL, Zuo X, Yang F, et al. Curcumin suppresses soluble tau dimers and corrects molecular chaperone, synaptic, and behavioral deficits in aged human tau transgenic mice. J Biol Chem 2013; 288: 4056-65.

[12] Park JS, Kim DH, Yoon SY. Regulation of amyloid precursor protein processing by its KFERQ motif. BMB Rep 2016; pii: 3370.

[13] Maiti P, Manna J, Veleri S, Frautschy S. Molecular chaperone dysfunction in neurodegenerative diseases and effects of curcumin. Biomed Res Int 2014; 2014: 495091.

[14] Macario AJL, Conway de Macario E, Cappello F. The Chaperonopathies. Diseases with Defective Molecular Chaperones. Dordrecht-Heidelberg-New York-London: Springer 2013.

[15] St George-Hyslop PH, Petit A. Molecular biology and genetics of Alzheimer's disease. C R Biol 2005; 328: 119-30.

[16] Chouraki V, Seshadri S. Genetics of Alzheimer's disease. Adv Genet 2014; 87: 245-94.

[17] Li Y, Bohm C, Dodd R, et al. Structural biology of presenilin 1 complexes. Mol Neurodegener 2014; 9: 59.

[18] Goedert M, Spillantini MG, Jakes R, Rutherford D, Crowther RA. Multiple isoforms of human microtubule-associated protein tau: sequences and localization in neurofibrillary tangles of Alzheimer's disease. Neuron 1989; 3: 519-26.

[19] Goedert M, Spillantini MG, Cairns NJ, Crowther RA. Tau proteins of Alzheimer paired helical filaments: abnormal phosphorylation of all six brain isoforms. Neuron 1992; 8: 159-68.

[20] Iwatsubo T, Odaka A, Suzuki N, Mizusawa H, Nukina N, Ihara Y. Visualization of A beta 42(43) and A beta 40 in senile plaques with end-specific A beta monoclonals: evidence that an initially deposited species is A beta 42(43). Neuron 1994; 13: 45-53.

[21] Braak H, Braak E. Neuropathological staging of Alzheimer-related changes. Acta Neuropathol 1991; 82: 239-59.

[22] Nelson PT, Braak H, Markesbery WR. Neuropathology and cognitive impairment in Alzheimer disease: a complex but coherent relationship. J Neuropathol Exp Neurol 2009; 68: 1-14.

[23] Nelson PT, Alafuzoff I, Bigio EH, et al. Correlation of Alzheimer disease neuropathologic changes with cognitive status: a review of the literature. J Neuropathol Exp Neurol 2012; 71: 362-81.

[24] Macario AJL, Conway de Macario E. Sick chaperones, cellular stress and disease. N Engl J Med 2005; 353: 1489-501.

[25] Macario AJL, Conway de Macario E. Chaperonopathies and chaperonotherapy. FEBS Lett 2007; 581: 3681-8.

[26] Gierasch LM. A career pathway in protein folding: from model peptides to post-reductionist protein science. Protein Sci 2011; 20: 783-90.

[27] Cappello F, Marino Gammazza A, Palumbo Piccionello A, et al. Hsp60 chaperonopathies and chaperonotherapy: targets and agents. Expert Opin Ther Targets 2014; 18: 185-208.

[28] Swerdlow RH. The neurodegenerative mitochondriopathies. J Alzheimer's Dis 2009; 17:737-51.

[29] Swerdlow RH, Burns JM, Khan SM. The Alzheimer's disease mitochondrial cascade hypothesis. J Alzheimers Dis 2010; 20: S265-79.

[30] Beck JS, Mufson EJ, Counts SE. Evidence for mitochondrial UPR gene activation in familial and sporadic Alzheimer's disease. Curr Alzheimer Res 2016; 13(6): 610-4.

[31] Perri ER, Thomas CJ, Parakh S, Spencer DM, Atkin JD. The unfolded protein response and the role of protein disulfide isomerase in neurodegeneration. Front Cell Dev Biol 2016; 3: 80.

[32] Ferri CP, Prince M, Brayne C, et al. Alzheimer's Disease International. Global prevalence of dementia: a Delphi consensus study. Lancet 2005; 366: 2112-7.

[33] Koren J 3rd, Jinwal UK, Lee DC, et al. Chaperone signalling complexes in Alzheimer's disease. J Cell Mol Med 2009; 13: 619-30.
[34] Neef DW, Jaeger AM, Thiele DJ. Heat shock transcription factor 1 as a therapeutic target in neurodegenerative diseases. Nat Rev Drug Discov 2001; 10: 930-44.

[35] Macario AJL, Conway de Macario E. Chaperonopathies by defect, excess, or mistake. Ann NYAcad Sci 2007; 1113: 178-91.

[36] Salbaum JM, Weidemann A, Lemaire HG, Masters CL, Beyreuther K. The promoter of Alzheimer's disease amyloid A4 precursor gene. EMBO J 1988; 7: 2807-13.

[37] Muchowski PJ, Wacker JL. Modulation of neurodegeneration by molecular chaperones. Nat Rev Neurosci 2005; 6:11-22.

[38] Satyal SH, Schmidt E, Kitagawa K, et al. Polyglutamine aggregates alter protein folding homeostasis in Caenorhabditis elegans. Proc Natl Acad Sci USA 2000; 97: 5750-55.

[39] Czarnecka AM, Campanella C, Zummo G, Cappello F. Mitochondrial chaperones in cancer: from molecular biology to clinical diagnostics. Cancer Biol Ther 2006; 5: 714-20.

[40] Bukau B, Horwich AL. The HSP70 and HSP60 chaperone machines. Cell 1998; 92: 351-66.

[41] Parnas A, Nisemblat S, Weiss C et al. Identification of elements that dictate the specificity of mitochondrial Hsp60 for its cochaperonin. PLoS One 2012; 7: e50318.

[42] Cappello F, Conway de Macario E, Di Felice V, Zummo G, Macario AJL. Chlamydia trachomatis infection and anti-Hsp60 immunity: the two sides of the coin. PLOS Pathog 2009; 5: e1000552.

[43] Cappello F, Conway de Macario E, Marasà L, Zummo G, Macario AJL. Hsp60 expression, new locations, functions and perspectives for cancer diagnosis and therapy. Cancer Biol Ther 2008; 7: 801-09.

[44] Barone R, Macaluso F, Sangiorgi C, et al. Skeletal muscle Heat shock protein 60 increases after endurance training and induces peroxisome proliferator-activated receptor gamma coactivator $1 \alpha 1$ expression. Sci Rep 2016; 6:19781.

[45] Vilasi S, Carrotta R, Mangione MR, et al. Human Hsp60 with its mitochondrial import signal occurs in solution as heptamers and tetradecamers remarkably stable over a wide range of concentrations. PLoS One 2014; 9: e97657.

[46] Spinello A, Ortore MG, Spinozzi F, et al. Quaternary structures of GroEL and naive-Hsp60 chaperonins in solution: a combined SAXS-MD study. RSC Adv 2015; 5: 49871-9.

[47] Chandra D, Choy G, Tang DG. Cytosolic accumulation of HSP60 during apoptosis with or without apparent mitochondrial release: evidence that its pro-apoptotic or pro-survival functions involve differential interactions with caspase-3. J Biol Chem 2007; 282: 31289-301.

[48] Arya R, Mallik M, Lakhotia S. Heat shock gene-integrating cell survival and death. J Biosci 2007; 32: 595-610.

[49] Shan YX, Liu TJ, Su HF, Samsamshariat A, Mestril R, Wang PH. Hsp10 and Hsp60 modulate Bcl-2 family and mitochondria apoptosis signaling induced by doxorubicin in cardiac muscle cells. J Mol Cell Cardiol 2003; 35: 1135-43.

[50] Merendino AM, Bucchieri F, Campanella C et al. Hsp60 is actively secreted by human tumor cells. PLoS One 2010; 5: e9247.

[51] Campanella C, Bucchieri F, Merendino AM, et al. The odyssey of Hsp60 from tumor cells to other destinations includes plasma membrane associated stages and Golgi and exosomal protein-trafficking modalities. PLoS One 2012; 7: e42008.

[52] Campanella C, Rappa F, Sciumè C, et al. Heat shock protein 60 levels in tissue and circulating exosomes in human large bowel cancer before and after ablative surgery. Cancer 2015; 121:3230-9.

[53] Gorska M, Marino Gammazza A, Zmijewski MA et al. Geldanamycin-induced osteosarcoma cell death is associated with hyperacetylation and loss of mitochondrial pool of heat shock protein 60 (hsp60). PLoS One 2013; 28: e71135.

[54] Campanella C, D'Anneo A, Gammazza AM, et al. The histone deacetylase inhibitor SAHA induces HSP60 nitration and its extracellular release by exosomal vesicles in human lung-derived carcinoma cells. Oncotarget 2015; [Epub ahead of print].

[55] Pace A, Barone G, Lauria A, et al. Hsp60, a novel target for antitumor therapy: structure-function features and prospective drugs design. Curr Pharm Des 2013; 19: 2757-64.

[56] Xanthoudakis S, Roy S, Rasper D, et al. HSP60 accelerates the maturation of procaspase- 3 by upstream activator proteases during apoptosis. EMBO J 1999; 18: 2049-56.

[57] Samali A, Cai J, Zhivotovsky B, et al. Presence of a pre-apoptotic complex of procaspase-3, HSP60 and HSP10 in the mitochondrial fraction of jurkat cells. EMBO J 1999; 18: 2040-8. 
[58] Cappello F, Conway de Macario E, Marino Gammazza A, et al. Hsp60 and human aging: Les liaisons dangereuses. Front Biosci (Landmark Ed) 2013; 18: 626-37.

[59] Hayflick L. The limited in vitro lifetime of human diploid cell strains. Exp Cell Res 1965; 37: 614-36.

[60] Raffetto JD, Leverkus M, Park HY, Menzoian JO. Synopsis on cellular senescence and apoptosis. J Vasc Surg 2001; 34: 173-7.

[61] Di Felice V, Ardizzone N, Marciano V, et al. Senescenceassociated HSP60 expression in normal human skin fibroblasts. Anat Rec A Discov Mol Cell Evol Biol 2005; 284: 446-53.

[62] Kaul Z, Yaguchi T, Kaul SC, Wadhwa R. Quantum dot-based protein imaging and functional significance of two mitochondrial chaperones in cellular senescence and carcinogenesis. Ann NY Acad Sci 2006; 1067: 469-73.

[63] Campanella C, Marino Gammazza A, Mularoni L, Cappello F, Zummo G, Di Felice V. A comparative analysis of the products of GROEL-1 gene from Chlamydia trachomatis serovar D and the HSP60 var1 transcript from Homo sapiens suggests a possible autoimmune response. Int J Immunogenet 2009; 36: 73-8.

[64] Marino Gammazza A, Bucchieri F, Grimaldi LM, Benigno A, Conway de Macario E, Macario AJL. The molecular anatomy of human Hsp60 and its similarity with that of bacterial orthologs and acetylcholine receptor reveal a potential pathogenetic role of antichaperonin immunity in myasthenia gravis. Cell Mol Neurobiol 2012, 32: 943-7.

[65] Binder RJ, Vatner R, Srivastava P. The heat-shock protein receptors: some answers and more questions. Tissue Antigens 2004; 64: 442-51.

[66] Pockley AG, Muthana M, Calderwood SK. The dual immunoregulatory roles of stress proteins. Trends Biochem Sci 2008; 33: 71-9.

[67] Gülden E, Märker T, Kriebel J, Kolb-Bachofen V, Burkart V, Habich C. Heat shock protein 60: evidence for receptor-mediated induction of proinflammatory mediators during adipocyte differentiation. FEBS Lett 2009; 583: 2877-81.

[68] Rodolico V, Tomasello G, Zerilli M, et al. Hsp60 and Hsp10 increase in colon mucosa of Crohn's disease and ulcerative colitis. Cell Stress Chaperones 2010; 15: 877-84.

[69] Tomasello G, Rodolico V, Zerilli M, et al. Changes in immunohistochemical levels and subcellular localization after therapy and correlation and colocalization with CD68 suggest a pathogenetic role of Hsp60 in ulcerative colitis. Appl Immunohistochem Mol Morphol 2011; 19: 552-61.

[70] Bellavia M, Tomasello G, Romeo M, et al. Gut microbiota imbalance and chaperoning system malfunction are central to ulcerative colitis pathogenesis and can be counteracted with specifically designed probiotics: a working hypothesis. Med Microbiol Immunol 2013; 202: 393-406.

[71] Barone R, Rappa F, Macaluso F, et al. Alcoholic liver disease: a mouse model reveals protection by Lactobacillus fermentum. Clin Transl Gastroenterol 2016; 7: e138.

[72] Cappello F, Caramori G, Campanella C, et al. Convergent sets of data from in vivo and in vitro methods point to an active role of Hsp60 in chronic obstructive pulmonary disease pathogenesis. PLoS One 2011; 6: e28200.

[73] Rizzo M, Macario AJL, Conway de Macario E, et al. Heat shock protein-60 and risk for cardiovascular disease. Curr Pharm Des 2011; 17: 3662-8

[74] D'Souza SM, Brown IR. Constitutive expression of heat shock proteins Hsp90, HSC70, Hsp70 and Hsp60 in neural and non-neural tissues of the rat during postnatal development. Cell Stress Chaperones 1998; 3:188-99.

[75] Stefano L, Racchetti G, Bianco F, et al. The surface-exposed chaperone, Hsp60, is an agonist of the microglial TREM2 receptor. J Neurochem 2009; 110: 284-94.

[76] Zhang D, Sun L, Zhu H, et al. Microglial LOX-1 reacts with extracellular HSP60 to bridge neuroinflammation and neurotoxicity. Neurochem Int 2012; 61: 1021-35.

[77] Stetler RA, Gan Y, Zhang W, et al. Heat shock proteins: cellular and molecular mechanisms in the central nervous system. Prog Neurobiol 2010; 92: 184-211.

[78] Marino Gammazza A, Colangeli R, Orban G, et al. Hsp60 response in experimental and human temporal lobe epilepsy. Sci Rep 2015; 5: 9434.

[79] McKenzie M, Lazarou M, Thorburn DR, Ryan MT. Analysis of mitochondrial subunit assembly into respiratory chain complexes using Blue Native polyacrylamide gel electrophoresis. Anal Biochem 2007; 364:128-37.

[80] Meriin AB, Sherman MY. Role of molecular chaperones in neurodegenerative disorders. Int J Hyperthermia 2005; 21: 403-19.

[81] Yoo BC, Kim SH, Cairns N, Fountoulakis M, Lubec G. Deranged expression of molecular chaperones in brains of patients with Alzheimer's disease. Biochem Biophys Res Commun 2001; 12: 24958 .

[82] Telling GC, Scott M, Mastrianni J, et al. Prion propagation in mice expressing human and chimeric PrP transgenes implicates the interaction of cellular PrP with another protein. Cell 1995; 83: 79-90.

[83] Jiang YQ, Wang XL, Cao XH, Ye ZY, Li L, Cai WQ. Increased heat shock transcription factor 1 in the cerebellum reverses the deficiency of Purkinje cells in Alzheimer's disease. Brain Res 2013; 26: 1519: $105-11$.

[84] Veereshwarayya V, Kumar P, Rosen KM, Mestril R, Querfurth HW. Differential effects of mitochondrial heat shock protein 60 and related molecular chaperones to prevent intracellular beta-amyloidinduced inhibition of complex IV and limit apoptosis. J Biol Chem 2006; 281: 29468-78.

[85] Choia J, Malakowskya CA, Talenta JM, et al. Anti-apoptotic proteins are oxidized by A“ $25-35$ in Alzheimer's fibroblasts. Biochim Biophys Acta 2003; 1637: 135-41.

[86] Boyd-Kimball D, Sultana R, Poon HF, et al. Proteomic identification of proteins specifically oxidized by intracerebral injection of amyloid beta-peptide (1-42) into rat brain: implications for Alzheimer's disease. Neuroscience 2005; 132: 313-24.

[87] Calabrese V, Sultana R, Scapagnini G, et al. Nitrosative stress, cellular stress response, and thiol homeostasis in patients with Alzheimer's disease. Antioxid Redox Signal 2006; 8: 1975-86.

[88] Nemirovsky A, Fisher Y, Baron R, Cohen IR, Monsonego A. Amyloid beta- HSP60 peptide conjugate vaccine treats a mouse model of Alzheimer's disease. Vaccine 2011; 23:4043-50.

[89] Walls KC, Coskun P, Gallegos-Perez JL. Swedish Alzheimer mutation induces mitochondrial dysfunction mediated by HSP60 mislocalization of amyloid precursor protein (APP) and beta-amyloid. J Biol Chem 2012; 31: 30317-27.

[90] Brocchieri L, Conway de Macario E, Macario AJL. Hsp70 genes in the human genome: Conservation and differentiation patterns predict a wide array of overlapping and specialized functions. BMC Evol Biol 2008; 8: 19.

[91] Mayer MP, Bukau B. Hsp70 chaperones: cellular functions and molecular mechanism. Cell Mol Life Sci 2007; 62. 670-84.

[92] Jiang J, Prasad K, Lafer EM, Sousa R. Structural basis of interdomain communication in the Hsc70 chaperone. Mol Cell 2005; 20: $513-24$.

[93] Scheufler C, Brinker A, Bourenkov G, et al. Structure of TPR domain-peptide complexes: critical elements in the assembly of the Hsp70-Hsp90 multichaperone machine. Cell 2000; 101: 199-210.

[94] Beere HM, Wolf BB, Cain K, Mosser DD, Mahboubi A, Kuwana $\mathrm{T}$, et al. Heat-shock protein 70 inhibits apoptosis by preventing recruitment of procaspase-9 to the Apaf-1 apoptosome. Nat Cell Biol 2000; 2: 469-75.

[95] Gao Y, Thomas JO, Chow RL, Lee GH, Cowan NJ. A cytoplasmic chaperonin that catalyses beta-actin folding. Cell 1992; 69: 104350 .

[96] Ferrari DM, Soling HD. The protein disulphide-isomerase family: unravelling a string of folds. Biochem J 1999; 339: 1-10.

[97] Beere HM: Stressed to death: regulation of apoptotic signalling pathways by the heat-shock proteins. Sci STKE 2001; 2001(93): re1.

[98] Rappa F, Farina F, Zummo G, et al. HSP-molecular chaperones in cancer biogenesis and tumor therapy: an overview. Anticancer Res 2012; 32: 5139-50.

[99] Zapun A, Darby NJ, Tessier DC, Michalak M, Bergeron JJ, Thomas DY. Enhanced catalysis of ribonuclease B folding by the interaction of calnexin or calreticulin with ER p57. J Biol Chem 1998; 273; 6009-12.

[100] Asea A, Rehli M, Kabingu E, et al. Novel signal transduction pathway utilized by extracellular HSP70: role of toll-like receptor (TLR) 2 and TLR4. J Biol Chem 2002; 277: 15028-34.

[101] Young RA. Stress proteins and immunology. Annu Rev Immunol 1990; 8: 401-20.

[102] Bonini NM. Chaperoning brain degeneration. Proc Natl Acad Sci USA 2002; 4: 16407-11. 
[103] Klucken J, Shin Y, Masliah E, Hyman BT, McLean PJ. Hsp70 reduces alpha-synuclein aggregation and toxicity. J Biol Chem 2004; 279: 25497-502.

[104] Njemini R, Abeele MV, Demanet C, Lambert M, Vandebosch S, Mets T. Age-related decrease in the inducibility of heat-shock protein 70 in human peripheral blood mononuclear cells. J Clin Immunol 2002; 22: 195-205.

[105] Ambra R, Mocchegiani E, Giacconi R, et al. Characterization of the hsp70 response in lymphoblasts from aged and centenarian subjects and differential effects of in vitro zinc supplementation. Exp Gerontol 2004; 39: 1475-84

[106] Magrané J, Smith RC, Walsh K, Querfurth HW. Heat shock protein 70 participates in the neuroprotective response to intracellularly expressed beta-amyloid in neurons. J Neurosci 2004; 24: 1700-6.

[107] Ali YO, Kitay BM, Zhai RG. Dealing with misfolded proteins: examining the neuroprotective role of molecular chaperones in neurodegeneration. Molecules 2010; 15: 6859-87.

[108] Hoshino T, Murao N, Namba T et al. Suppression of Alzheimer's disease-related phenotypes by expression of heat shock protein 70 in mice. J Neurosci 2011; 31: 5225-34.

[109] Evans CG, Wisen S, Gestwicki JE. Heat shock proteins 70 and 90 inhibit early stages of amyloid beta-(1-42) aggregation in vitro. J Biol Chem 2006; 281: 33182-91.

[110] Jinwal UK, Akoury E, Abisambra JF, et al. Imbalance of Hsp70 family variants fosters tau accumulation. FASEB J 2013; 27: 14509.

[111] Voss K, Combs B, Patterson KR, Binder LI, Gamblin TC. Hsp70 alters tau function and aggregation in an isoform specific manner. Biochemistry 2012; 51: 888-98.

[112] Kawakami F, Suzuki M, Shimada N, et al. Stimulatory effect of $\alpha-$ synuclein on the tau-phosphorylation by GSK-3 $\beta$. FEBS J 2011; 278: 4895-904.

[113] Elliott E, Tsvetkov P, Ginzburg I. BAG-1 associates with Hsc70.Tau complex and regulates the proteasomal degradation of Tau protein. J Biol Chem 2007; 282: 37276-84.

[114] Abisambra J, Jinwal UK, Miyata Y, et al. Allosteric heat shock protein 70 inhibitors rapidly rescue synaptic plasticity deficits by reducing aberrant tau. Biol Psychiatry 2013; 74: 367-74.

[115] Wandinger SK, Richter K, Buchner J. The Hsp90 chaperone machinery. J Biol Chem 2008; 4; 283: 18473-7.

[116] Csermely P, Schnaider T, Soti C, Prohaszka Z, Nardai G. The 90$\mathrm{kDa}$ molecular chaperone family: structure, function, and clinical applications. A comprehensive review. Pharmacol Ther 1998; 79: 129-68.

[117] Jakob U, Meyer I, Bugl H, Andre S, Bardwell JC, Buchner J. Structural organization of procaryotic and eucaryotic Hsp90. Influence of divalent cations on structure and function. J Biol Chem 1995; 270: 14412-9.

[118] Rappa F, Sciume C, Lo Bello M, et al. Comparative analysis of Hsp10 and Hsp90 expression in healthy mucosa and adenocarcinoma of the large bowel. Anticancer Res 2014; 34: 4153-9.

[119] Bagatell R, Whitesell L. Altered Hsp90 function in cancer: a unique therapeutic opportunity. Mol Cancer Ther 2004; 3:1021-30.

[120] Thomas M, Harrell JM, Morishima Y, Peng HM, Pratt WB, Lieberman AP. Pharmacologic and genetic inhibition of Hsp90dependent trafficking reduces aggregation and promotes degradation of the expanded glutamine androgen receptor without stress protein induction. Hum Mol Genet 2006; 15: 1876-83.

[121] Luo W, Dou F, Rodina A, et al. Roles of heat shock protein 90 in maintaining and facilitating the neurodegenerative phenotype in tauopathies. Proc Natl Acad Sci USA 2007; 104: 9511-6.

[122] Ansar S, Burlison JA, Hadden MK, et al. A non-toxic Hsp90 inhibitor protects neurons from Abeta-induced toxicity. Bioorg Med Chem Lett 2007; 17: 1984-90.

[123] Ou JR, Tan MS, Xie AM, Yu JT, Tan L. Heat shock protein 90 in Alzheimer's disease. Biomed Res Int 2014; 2014: 796869.

[124] Kakimura JI, Kitamura Y, Takata K, et al. Microglial activation and amyloid- $\beta$ clearance induced by exogenous heat-shock proteins. FASEB J 2002; 16: 601-3.

[125] Sahara N, Maeda S, Yoshiike Y, et al. Molecular chaperonemediated tau protein metabolism counteracts the formation of granular tau oligomers in human brain. J Neurosci Res 2007; 85: 3098-108.

[126] Thompson AD, Scaglione KM, Prensner J, et al. Analysis of the tau-associated proteome reveals that exchange of Hsp70 for Hsp90 is involved in tau degradation. ACS Chem Biol 2012; 7: 1677-86.

[127] Zhao H, Michaelis ML, Blagg BSJ. Hsp90 modulation for the treatment of Alzheimer's disease. Adv Pharmacol 2012; 64: 1-25.

[128] Pulliam L, Gupta A. Modulation of cellular function through immune-activated exosomes. DNA Cell Biol 2015; 34: 459-63.

[129] Takeuchi T, Suzuki M, Fujikake N, et al. Intercellular chaperone transmission via exosomes contributes to maintenance of protein homeostasis at the organismal level. Proc Natl Acad Sci USA 2015; 112: E2497-506. 